\title{
Changes in Resting Energy Expenditure following Gastric Bypass Surgery: Impact on Total Body Weight.
}

\section{Abstract}

Bariatric surgery, including Gastric Bypass (GBP) Surgery, is the most efficient modality to manage severe obesity. Resting Energy Expenditure (REE) is an area of interest in the context of weight loss (WL) as it has been postulated to be an independent predictor of WL success following GBP. As such, the aim of this study is to investigate the impact of REE on WL following GBP. 31 GBP patients $(77.4 \%$ females BMI $45.5 \pm 7.0 \mathrm{~kg} / \mathrm{m} 2 ; 47.3 \pm 11.6 \mathrm{y})$ and 32 weight-stable controls $(46.9 \%$ females; $\mathrm{BMI} 27.0 \pm 4.6 \mathrm{~kg} / \mathrm{m} 2 ; 41.1 \pm$ $13.5 \mathrm{y})$ were assessed at one-month pre-surgery and at 3 and 12-months post-surgery. Fat mass (FM) and fat-free mass (FFM) were measured using dual energy X-ray absorptiometry (Lunar iDXA, GE Healthcare). REE was measured under standardised conditions using indirect calorimetry (ECAL, Metabolic Health Solutions). Statistical analyses were performed with SPSS v24.0, Armonk, NY. Multiple regression analysis showed that FM $(\mathrm{P}=0.001)$, FFM $(\mathrm{P}<0.0001)$ and gender $(P=0.012)$ significantly predicted the interindividual variability in REE. Total body weight (TBW) was removed from the model due to collinearity. Adjusted-REE values were then generated using the above predictor variables. Low-REE and high-REE groups were created using within-group adjusted-REE split. At both follow-ups (3- and 12-months post-surgery), patients had a greater reduction in TBW, FM, FFM, measured-REE and adjusted-REE values compared with controls $(P<0.0001)$. There was also no significant difference between measured and adjusted-REE values at all time-points $(P>0.05)$. Patients with high REEs at baseline lost more TBW than those in the low-REE group at 3-months post-surgery $(-24.9 \pm 6.5 \mathrm{~kg}$ vs. $-16.6 \pm 7.0 \mathrm{~kg} ; \mathrm{P}=0.005)$ and 12 -months post-surgery $(-41.3 \pm 12.5 \mathrm{~kg}$ vs. $-25.8 \pm 10.4 .0 \mathrm{~kg} ; \mathrm{P}=0.003)$. There was no significant difference in mean $\mathrm{TBW}$ changes for controls in the low-and high-REE groups at both follow-ups. Patients with high REEs at 3-months post-surgery did not lose more TBW than those in the low-REE group at 12 -months post-surgery $(-30.1 \pm 12.8 \mathrm{~kg}$ vs. $-38.6 \pm 14.4 \mathrm{~kg} ; \mathrm{P}=0.155)$. Similarly, there was no difference in mean TBW reduction between controls in the low- and high-REE groups $(\mathrm{P}=0.115)$. Thus while patients with a high adjusted-REE value at baseline ( $>9746.6 \mathrm{~kJ} /$ day) lost more weight at 3- and 12-months post-GBP, it is plausible that from the third to the 12th month post-surgery, other key drivers of weight loss, particularly the reduction in energy intake are more important in predicting WL. Further research with a larger sample size is required to increase the chances of detecting a true effect.

\section{Conflict of Interest}

There is no conflict of interest 